\title{
Ácido acetilalicílico associado ao cálcio na prevenção da pré-eclâmpsia em gestantes hipertensas crônicas selecionadas pela dopplervelocimetria das artérias uterinas
}

\section{Aspirin and calcium to prevent preeclampsia in chronic hypertensive women with abnormal uterine artery Doppler ultrasound}

Autor: Eder Viana de Souza.

Orientador: Prof. Dr. Nelson Sass.

Tese de Doutorado apresentada ao Departamento de Obstetrícia da Universidade Federal de São Paulo - Escola Paulista de Medicina, em 30 de agosto de 2005.

Objetivo: estudar a associação de baixas doses de ácido acetilsalicílico ( AAS) e Cálcio ( $2 \mathrm{~g} /$ dia) na prevenção da pré-eclâmpsia $(\mathrm{PE})$, em gestantes hipertensas crônicas, selecionadas através da dopplervelocimetria das artérias uterinas. Analisamos também a efetividade da dopplervelocimetria para predição da PE. Métodos: estudo aleatório, controlado e duplo cego. 91 gestantes foram submetidas a dopplervelocimetria entre a $20^{\mathrm{a}}$ e $28^{\mathrm{a}}$ semanas de gestação. As 42 gestantes com dopplervelocimetria normal $(46,1 \%)$ não receberam medicação. As 49 pacientes com indice de resistência elevado e/ou incisura protodiastólica foram alocadas aleatoriamente para o grupo placebo $(28,5 \%)$ ou no grupo tratado $(25,2 \%)$. Resultados: a dopplervelocimetria foi efetiva para predição de PE $(p=0,001)$, de prematuridade $(p=0,014)$ e restrição de crescimento intra-uterino (RCIU). A comparação entre o grupo placebo e grupo tratado não demonstrou diferenças quanto a $\mathrm{PE}(\mathrm{p}=0,74)$, e prematuridade $(p=0,99)$. Ocorreu redução significativa na freqüência de RCIU a favor da medicação $(p=0,098)$. Conclusões: a dopplervelocimetria foi efetiva para a predição da PE de suas complicações. Nossos resultados não permitem apoiar o uso de AAS, associada ao cálcio, em gestantes hipertensas crônicas selecionadas pela dopplervelocimetria das artérias uterinas, na prevenção da pré-eclâmpsia. Foi observada uma redução significante na freqüência de RCIU a favor da utilização das medicações associadas.

PALAVRAS-CHAVE: Prevenção; Pré-eclâmpsia; Ácido acetilsalicílico; Cálcio; Dopplervelocimetria

Resumo de Tese

\section{Prevalência de complicações obstétricas em parturientes adolescentes}

\section{Prevalence of obstetric complications in adolescent women in labor}

Autor: Jacio Berardo Sampaio

Orientador: Prof. Dr. Fabrício da Silva Costa

Dissertação apresentada ao Curso de Mestrado Profissional em Saúde da Criança e do Adolescente da Universidade Estadual do Ceará (UECE), para obtenção do título de Mestre em Saúde da Criança e do Adolescente, em 11 de dezembro de 2005.

Objetivo: determinar a prevalência de complicações obstétricas em parturientes adolescentes, identificando os tipos de complicações obstétricas e perinatais mais freqüentes e avaliar as vias de parto em hospitais terciários da região do Cariri-Ce, entre junho de 2004 a maio de 2005. Metodologia: trata-se de um estudo transversal cuja abordagem é quantitativa. Os dados foram obtidos dos prontuários das parturientes e de entrevista estruturada, especialmente elaborada para esta pesquisa, considerando-se as variáveis sócio-demográficas, psicológicas, biológicas referentes à puérpera e ao recém-nascido. Para processar as informações, foram utilizados o sistema Epi-info processador de texto $6.04 \mathrm{a}$, banco de dados e estatística para epidemiologia. A associação entre variáveis foi testada pelo $\chi^{2}$ ou pelo Teste Exato de Fisher, com nivel de significância de 5\%. Resultados: foram analisadas 1321 gestantes adolescentes com média de ida- de de 17,3 anos. A idade mínima foi de 11 e a máxima de 19 anos, e a grande maioria 95,7\% eram de adolescentes tardias (15 a 19 anos). Quanto ao tipo de parto $56 \%$ foram de partos vaginais e $44 \%$ de partos cesarianas. Em relação à prevalência de complicações obstétricas $22 \%$ apresentaram algum tipo de complicação, sendo as mais freqüentes, pré-eclâmpsia (5\%), rotura prematura das membranas $(4,5 \%)$, sofrimento fetal $(3,6 \%)$ e parto pós-termo $(2,1 \%)$. Conclusão: é importante conhecer a prevalência de complicações obstétricas, os tipos de complicações e as vias de parto nas parturientes adolescentes em cada região, a fim de que estratégias possam ser adotadas para minimizar as repercussões desfavoráveis e direcionar as ações preventivas.

PALAVRAS-CHAVE: Adolescência; Gravidez; Complicações; Cesariana 